\title{
Multi-Point Aerodynamic Shape Optimization of Rotor Blades Using Unstructured Meshes
}

\author{
Sang Wook Lee* and Oh Joon Kwon** \\ Korea Advanced Institute of Science and Technology \\ 373-1, Guseong-dong Yuseong-gu, Daejeon, 305-701, Korea
}

\begin{abstract}
A multi-point aerodynamic shape optimization technique has been developed for helicopter rotor blades in hover based on a continuous adjoint method on unstructured meshes. The Euler flow solver and the continuous adjoint sensitivity analysis were formulated on the rotating frame of reference. The objective function and the sensitivity were obtained as a weighted sum of the values at each design point. The blade section contour was modified by using the Hicks-Henne shape functions. The mesh movement due to the blade geometry change was achieved by using a spring analogy. In order to handle the repeated evaluation of the design cycle efficiently, the flow and adjoint solvers were parallelized based on a domain decomposition strategy. A solution-adaptive mesh refinement technique was adopted for the accurate capturing of the wake. Applications were made to the aerodynamic shape optimization of the Caradonna-Tung rotor blades and the $\mathrm{UH}-60$ rotor blades in hover.
\end{abstract}

Key Words : Multi-point Aerodynamic Shape Optimization, Rotor Blades, Hover, Unstructured Meshes

\section{Introduction}

Aerodynamic shape optimization has been one of the most important research areas in computational fluid dynamics, and it enables aircraft designers to determine optimum aerodynamic configurations. In the early days of the development, its application was mostly restricted to simple two-dimensional geometries due to the lack of sufficient computing power. However, recent advancement in the computer hardware technology and the numerical algorithms made its applications to more complex three-dimensional configurations possible [1]. Currently, several variations of the aerodynamic shape optimization technique exist. Among them, one of the most popular methods available is the gradient-based optimization technique in which sensitivities, the gradients of the objective function with respect to design variables, are used to update the design variables in the direction that the specified objective function is minimized.

Several researches were previously conducted for the efficient evaluation of the sensitivities. Recently, particular interest has been given to adjoint methods, in which sensitivities are calculated indirectly from the single evaluation of the flow and adjoint equations. Since the computational time for solving the adjoint equations is similar to that of the flow equations and is independent to the number of design variables, adjoint methods are widely used for the aerodynamic shape optimization of three-dimensional configurations requiring a large number of design variables [2].

* Graduate Research Assistant, Aerospace Engineering

** Corresponding author, Professor, Aerospace Engineering

E-mail:ojkwon@kaist.ac.kr Tel:042-869-3722 Fax : 042-869-3710 
Shock waves in transonic flow not only increase the drag but also cause unfavorable flutter or buffet to fixed wings. Helicopter rotor blades in forward flight are also frequently exposed to strong unsteady shock waves at the blade tip region. These shock waves increase the required power, and become a source of undesirable pitch-down moment, noise and vibration. Thus, elimination or possible reduction of the strength of these shock waves is desirable for the enhancement of the helicopter performance. However, application of the aerodynamic shape optimization techniques has been mostly limited to fixed-wing configurations, and the aerodynamic design of helicopter rotor blades has not been benefited much from the current optimization techniques. Since the flow field of rotor blades is very complex, inherently unsteady, and highly three-dimensional, the application of aerodynamic design optimization techniques to rotor blades is much more challenging than that to fixed wings.

Currently, only a limited number of researches were conducted for the application of the aerodynamic shape design technique to helicopter rotor blades. Tapia, Sankar, and Schrage [3] developed an inverse design method for rotor blades based on the modified Garabedian-McFadden technique and a transonic full-potential flow analysis. From the prescribed target pressure distributions, blade section contours at a few spanwise control stations were designed in forward flight by using a multi-point design technique. Sun et al. [4] studied the aerodynamic design of helicopter rotor blades in forward flight by using a response surface method coupled with a genetic algorithm based on a free-wake panel method. However, this technique requires a very large number of flow calculation, and thus the extension to high fidelity flow physics or complex geometries requiring a large number of computational cells is still a formidable task. Recently, Lee and Kwon [5] developed an aerodynamic shape optimization technique for helicopter rotor blades in hover using a continuous adjoint method. However, the applications were made to a specified single-point operating condition, while helicopter rotor blades operate at varying tip Mach number and collective pitch angle. The aerodynamic performance of the optimized configuration for a specific design condition may degrade at off-design operating conditions. Thus, it is important to incorporate a multi-point design optimization capability [6] when the shape optimization is performed for helicopter rotor blades.

In the present study, a multi-point aerodynamic shape optimization technique has been developed for helicopter rotor blades in hover based on the previously developed singlepoint shape optimization technique [5]. For this purpose, the sensitivity and the objective function were obtained as a weighted sum of those at each individual design point. The airfoil section contour was represented by using the Hicks-Henne shape functions. Mesh movement due to the changing blade geometry was handled by using a spring analogy. A solution-adaptive mesh refinement technique was applied for the accurate capturing of the wake. In order to handle the large computational time involved in the repeated evaluation of the flow and adjoint solvers, a parallel algorithm based on domain decomposition was adopted Validations were made for the aerodynamic shape optimization of the Caradonna-Tung experimental rotor blade [7] and the $\mathrm{UH}-60$ rotor blade [8] in hover.

\section{Numerical Method}

\section{Flow analysis}

The equations governing three-dimensional, steady, inviscid, compressible flows are the Euler equations, which can be recast for absolute flow variables on a rotating frame of reference for rotors in hover. The governing equations are discretized by using a finitevolume method [9]. The inviscid flux across each cell face is computed based on the Roe's flux-difference splitting formula. To obtain second-order spatial accuracy, estimation of the state variables at each cell face is achieved by interpolating the solution using the 
Taylor series expansion in the neighborhood of each cell center. The cell-averaged solution gradient required at the cell center for the above expansion is computed from the Gauss' theorem by evaluating the surface integral for the closed surface of the tetrahedron [10]. The nodal value of the solution required by the above expansion is computed from the surrounding cell center data using a second-order accurate pseudo-Laplacian averaging procedure [11].

An implicit time integration algorithm based on the linearized Euler backward differencing is used to advance the solution in time. The linear system of equations is solved at each time step using a point Gauss-Seidel method.

On the solid surface of the rotor blade, the flow tangency condition is applied. The grid velocity due to rotation is also accounted for at the solid surface. At the far-field boundary, a source-sink boundary condition proposed by Srinivasan, Raghavan, and Duque [12] is used. Due to the periodic nature of the flow of hovering rotors, calculations are made for a single blade, and the periodic boundary condition is imposed between blades.

\section{Sensitivity analysis}

The aerodynamic shape optimization problem for rotor blades can be stated as the minimization of the objective function consisted of the aerodynamic performance parameters such as thrust, torque, or combination of the two. The problem is also subject to the governing flow equations. This non-linear constraint optimization problem can be recast to an unconstraint optimization, which is more efficient and easy to apply. This is done by setting a new objective function represented as the combination of the original objective function and the flow equations by introducing the Lagrangian multipliers (adjoint variables). The continuous adjoint equations, the adjoint boundary conditions, and the adjoint sensitivities can be derived by taking the variation of the new objective function with respect to the design variables [5].

The linear adjoint equations of the Euler equations can be discretized by using any stable method. However, the discretization must be consistent with that of the flow equations for the accurate evaluation of the sensitivity. In the present study, the adjoint flux was calculated using a second-order accurate upwind scheme, which is similar to that of the flow equations [5]. Since the mathematical characteristics of the adjoint equations are identical to those of the flow equations, most of the subroutines developed for the flow solver can be used for solving the adjoint equations with little modification.

\section{Objective function}

The main objective of the present study is to obtain rotor blade configurations which minimize the required inviscid torque under the constraint of maintaining the desired thrust level. The objective function selected in the present study is a combination of the thrust and torque coefficients:

$$
I=\sum_{i=1}^{n d p} C_{i}\left[w_{1}\left(C_{T, r e q}-C_{T}\right)^{2}+w_{2}\left(C_{Q}\right)^{2}\right]_{i}
$$

Here $n d p$ is the number of design points, and $C_{i}$ is the weighting factor selected by the designer by considering the importance of each individual design point. $w_{1}$ and $w_{2}$ are the penalty constants set by the designer and determine the relative importance of each term for the given design problem of interest. However, large difference between the two weighting coefficients may increase the stiffness of the design problem and the design 
process may fail as a result. In the present study, $w_{1}$ and $w_{2}$ are set to 0.5 and 2.5 , respectively, and $C_{i}$ is set to one for all design cases.

\section{Shape function and mesh deformation}

During the optimization process, the blade geometry changes continuously by following the gradient information. Ideally, all nodes on the blade surface can be used as the design variables. However, to avoid the numerical error involved in the gradient information and to reduce the large calculation time for evaluating the mesh sensitivities, a proper surface parameterization must be used. In the present study, the Hicks-Henne shape functions [13] are used to reduce the number of design variables and to represent the blade section contours smoothly. The Hicks-Henne shape functions are composed of exponential and sine bumps, and have been proven to be effective in achieving design improvement, even using a small number of design variables. Then, the blade geometry can be represented as

$$
y=y_{o}+\sum_{j=1}^{n d v} \mathbf{d}_{j} f_{j}
$$

where $y, y_{o}, \mathbf{d}_{j}, f_{j}$ and $n d v$ represent the new coordinate after the design, the original geometry, the vector of design variables, the shape functions, and the number of design variables, respectively.

During the design iteration, the computational mesh deforms to reflect the change of the blade section contour. In the present study, the mesh movement is treated by using a spring analogy [14].

\section{Parallel implementation}

In order to reduce the large computational time involved in the repeated evaluation of the flow and adjoint solvers, a parallel algorithm based on the domain decomposition strategy is applied. The load balancing between processors is achieved by partitioning the global computational domain into local sub-domains using the MeTiS libraries. The Message Passing Interface is used to transfer the flow and adjoint variables across the sub-domain boundaries. No attempt is made to parallelize the optimization algorithm since this portion is computationally negligible compared to the other elements of the design process.

All calculations are made on a $\mathrm{PC}$-based Linux cluster having $2.4 \mathrm{GHz}$ Pentium IV CPUs.

\section{Solution-adaptive mesh refinement}

The aerodynamic performance of helicopter rotor blades in hover is strongly affected by their own wake lingering underneath the rotor disk plane. In order to preserve the tip vortex and to reflect its effect on the present blade shape optimization accurately, a multilevel solution-adaptive mesh refinement technique is adopted [9].

At first, the flow calculation is made on the coarse mesh. When the solution converges, the calculation is paused, and the location of local vorticity maximum is searched at every five degrees of the vortex age from the blade tip. This search continues until the tip vortex core cannot be identified due to the numerical dissipation. Next, the discrete vortex cores are connected to form a smooth line using a three-dimensional parabolic blending. Then 1:8 division is applied to cells on and near the captured core trajectory by inserting new nodes in the middle of tetrahedral cell edges. Buffer cells are also used between the divided cells and the surrounding cells to confirm valid cell connectivity. These buffer cells are divided using either $1: 4$ or 1:2 division patterns. Then, new domain decomposition is applied to the 
refined mesh and the calculation resumes. The above process is repeated to achieve a multi-level mesh refinement as needed.

\section{Datatiled design process}

In the process of the multi-point shape optimization, the weighted sum of the objective function and the sensitivities at each design point is used to advance the design iteration.

Initially, a coarse mesh is constructed for a specified reference design point and the given rotor geometry. Next, the flow calculation is made, and the multi-level solutionadaptive mesh refinement is simultaneously applied. Then, the meshes for the remaining design points are constructed by properly moving the mesh points from the refined mesh using the spring analogy. The flow and adjoint solutions are calculated for all design points, and the objective function and the sensitivities are evaluated. Then, the design variables are updated as

$$
\mathbf{d}^{n+1}=\mathbf{d}^{n}+\lambda \mathbf{s}^{n}
$$

where $\mathbf{S}$ is the search direction obtained as a weighted sum of the gradients for all design points. $\lambda$ is the step size, and $\mathbf{d}$ is the design variables. In the present study, the steepest descent algorithm is used by assigning $\lambda$ as a constant. Once this design cycle is completed, the meshes are modified to cope with the changes in the blade geometry, and the flow and adjoint solutions are calculated for the next design cycle. This optimization process ends when the reduction rate of the objective function value becomes sufficiently small or when the search direction cannot be identified for further reducing the objective function value.

\section{Results and Discussion}

\section{Caradonna-Tung rotor blade}

At first, the present method was applied to the Caradonna-Tung experimental rotor configuration [7]. The rotor has two untwisted blades, which are made of a NACA0012 airfoil section and have a rectangular planform shape with an aspect ratio of six. The blade pre-cone angle was set to 0.5 degrees.

The design conditions selected for the present study were three collective pitch angles 7, 8 , and 9 degrees, and three transonic tip Mach numbers, 0.833, 0.877, and 0.921. Three multi-point shape optimization cases were studied by combining the selected tip Mach numbers and collective pitch angles. As the first design case, the rotor operating condition was set to a tip Mach number of 0.877 and the collective pitch angle varied from 7 to 9 degrees. The second case was studied at 8 degree collective pitch angle and three tip Mach numbers, 0.833, 0.877, and 0.921. The last case was studied for three different tip Mach number and collective pitch angle combinations; i) tip Mach number 0.921 and 7 degree collective pitch angle, ii) tip Mach number 0.877 and 8 degree collective pitch angle, and iii) tip Mach number 0.833 and 9 degree collective pitch angle.

Figure 1 (a) shows the surface triangulations at the periodic boundary and at the farfield boundary located at two radii away from the rotor in all directions. The grid periodicity at the periodic boundary between the blades can be confirmed in this figure. In Fig. 1(b), the surface triangulations on the rotor blade are shown. The computational domain was initially divided by 314,417 tetrahedral cells and 58,169 nodes. The bold lines represent the parallel sub-domain boundaries.

In order to reduce the numerical dissipation and to preserve the tip vortex more accurately, five levels of mesh adaptation were applied to the wake. The cells tagged for 
refinement along the tip vortex were divided until the characteristic length of the cell became less than $5 \%$ of the blade chord length. The final mesh after the mesh refinement consisted of 563,073 cells and 101,014 nodes. The mesh adaptation sequence for wake capturing is presented in Fig. 2. After each level of mesh adaptation, the refined mesh was re-partitioned for load balancing.

Initially, validation of the flow solver was made by calculating the flow at the tip Mach number of 0.877 and the collective pitch angle of 8 degrees. Figure 3 shows the comparison of the surface pressure distributions at several spanwise stations of the original CaradonnaTung rotor blade. It shows that the predicted surface pressure distributions are generally in good agreement with the experiment at all spanwise stations. After the mesh refinement, the shock strength increased slightly and the shock wave is located at further downstream from that of the initial mesh. This is due to the enhanced interaction between the current blade and the tip vortex from the preceding blade after mesh adaptation.



a) Surface triangulations at the computational boundary

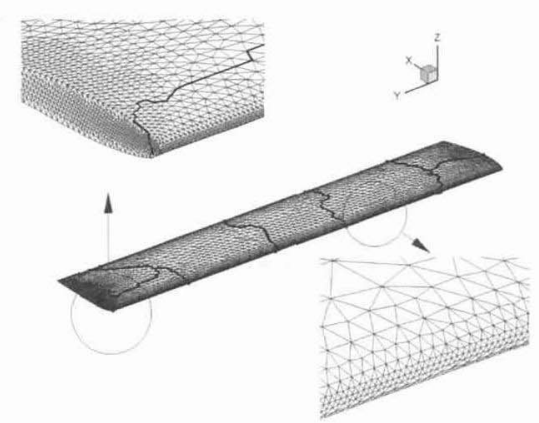

b) Surface triangulations on the rotor blade

Fig. 1. Computational mesh for the Caradonna-Tung rotor blade



a) First-level adaptation



d) Fourth-level adaptation

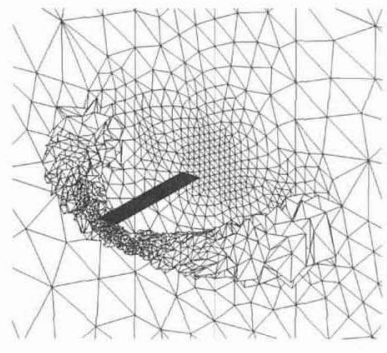

b) Second-level adaptation

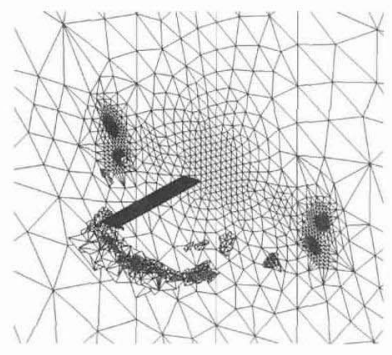

e) Fifth-level adaptation

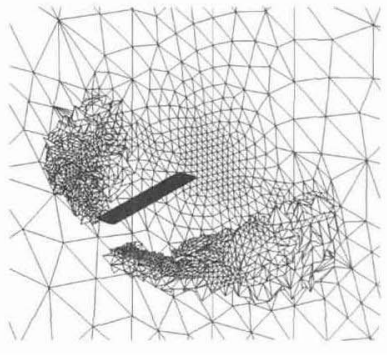

c) Third-level adaptation

\begin{tabular}{|c|c|c|}
\hline & ncell & nnode \\
\hline Initial & 314,417 & 58,169 \\
\hline 1 level & 342,867 & 63,088 \\
\hline 2 level & 436,776 & 79,143 \\
\hline 3 level & 527,300 & 94,710 \\
\hline 4 level & 553,943 & 99,424 \\
\hline 5 level & 563,073 & 101,014 \\
\hline
\end{tabular}

Fig. 2. Wake mesh adaptation sequence for the Caradonna-Tung rotor 


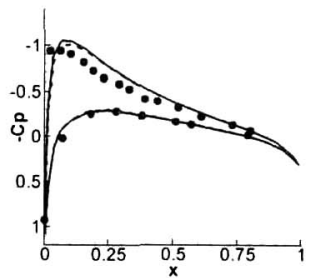

a) $r / R=0.68$

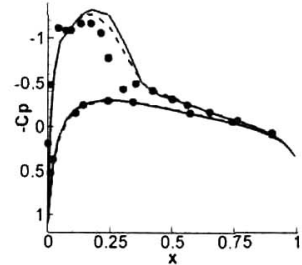

b) $r / R=0.80$



c) $r / R=0.89$

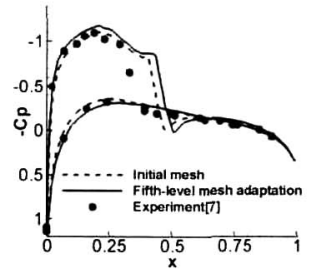

d) $r / R=0.96$

Fig. 3. Comparison of the surface pressure distributions of the Caradonna-Tung rotor blade

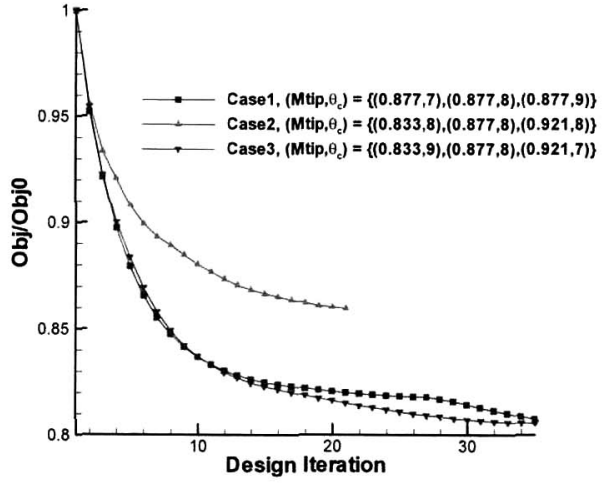

Fig. 4. Comparison of convergence histories

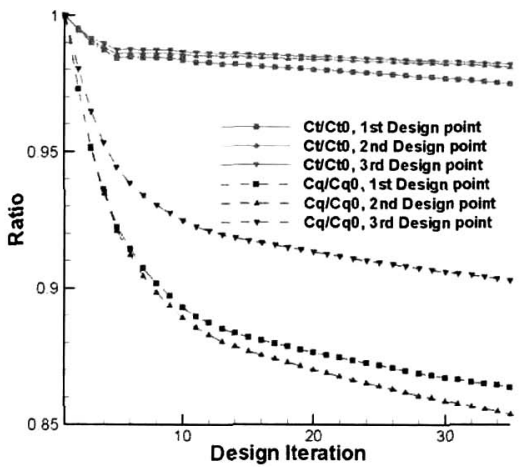

Fig. 5. Convergence history for the design case 3

Figure 5 shows the convergence history of the rotor thrust and the inviscid torque for the design case 3 . After 36 design iterations, the inviscid torque was reduced by approximately $10 \%$ to $15 \%$ from the initial values while the thrust losses were less than $2 \%$. The total calculation time required for the optimization was approximately seven hours using 24 processors.

In Figs. 6, 7, and 8, the surface pressure distributions at four spanwise stations of the blade before and after the design optimization are compared for each design point of the design case 3 . It is shown that the shock wave on the original Caradonna-Tung rotor blade mostly disappeared at the inboard sections. At the outboard sections, even though the shock wave was not completely eliminated as typically known for three-dimensional wings, the shock strength was significantly reduced after the design optimization, particularly at the design points with high tip Mach numbers.



a) $r / R=0.82$

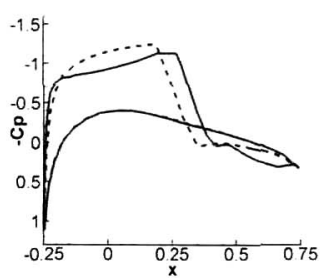

b) $r / R=0.87$

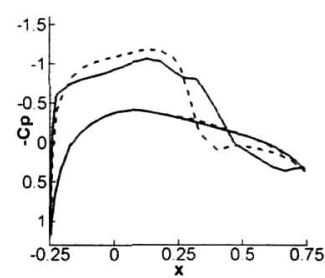

c) $r / R=0.92$

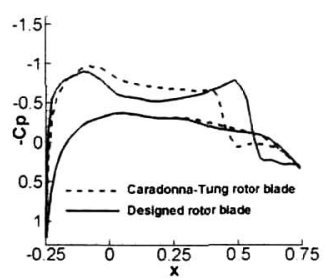

d) $r / R=0.98$

Fig. 6. Comparison of the surface pressure distributions at the first design point of case 3 


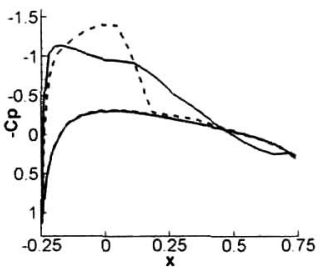

a) $r / R=0.82$

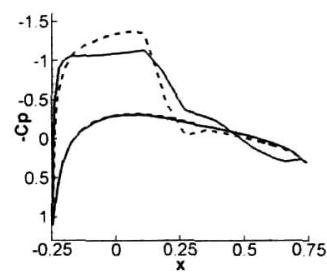

b) $r / R=0.87$



c) $r / R=0.92$

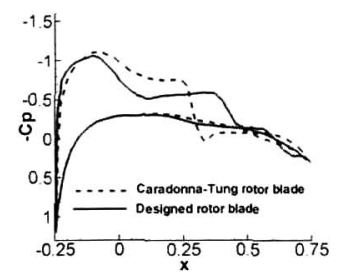

d) $r / R=0.98$

Fig. 7. Comparison of the surface pressure distributions at the second design point of case 3

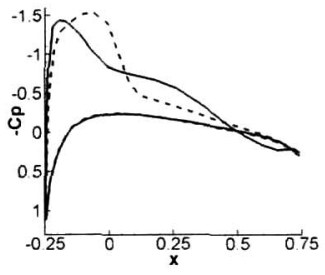

a) $r / R=0.82$

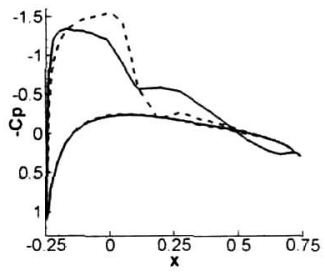

b) $r / R=0.87$

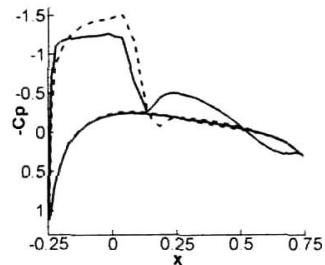

c) $r / R=0.92$

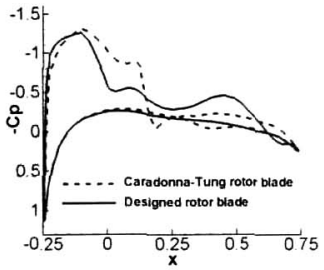

d) $r / R=0.98$

Fig. 8. Comparison of the surface pressure distributions at the third design point of case 3

In Fig. 9, the blade section contours at four spanwise stations near the blade tip are compared between the original and designed rotors. It is shown that the leading-edge radius and the blade thickness near the leading edge were slightly reduced for all design sections. The blade thickness gradually increases at and downstream of the mid chord, particularly at further outboard sections.

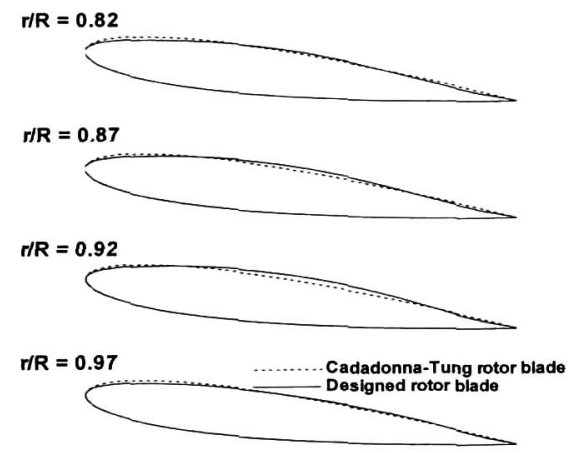

Fig. 9. Comparison of the blade section contours of the Caradonna-Tung rotor blade before and after design

In table 1, the calculated thrust, torque and pitching moment are compared between the original Caradonna-Tung rotor blade and the blade designed from case 3. It is observed that, after the optimization, the nose-up pitching moment was slightly reduced for all design points. This is due to the higher aft-loading as shown in Figs. 6, 7, and 8 obtained as a result of the blade thickness increase.

The off-design performance of the designed rotor blade from case 3 was tested at a subsonic tip Mach number of 0.439 and a collective pitch angle of 8 degrees. In Fig. 10 , comparison of the blade surface pressure distributions before and after the design is presented. It shows that the loading was slightly reduced near the leading edge due to the 
loss in the blade thickness. However, slightly higher loading was obtained at the mid chord because of the flow acceleration caused by the thickness increase. As a result, the total thrust obtained from the designed rotor blade was approximately $98 \%$ of that of the original blade. This demonstrates that reasonable performance can be obtained from the designed rotor at off-design subsonic operating conditions.

Table 1. Comparison of aerodynamic coefficients of the Caradonna-Tung rotor before and after design

\begin{tabular}{|l|c|ccc|}
\hline \multicolumn{2}{|c|}{ CASE 3 } & Ct & $\mathrm{Cq}$ & $\mathrm{Cm}$ \\
\hline Design & Before & $5.778 \mathrm{E}-03$ & $6.498 \mathrm{E}-04$ & $1.376 \mathrm{E}-05$ \\
Point 1 & After & $5.634 \mathrm{E}-03$ & $5.613 \mathrm{E}-04$ & $2.493 \mathrm{E}-06$ \\
\hline Design & Before & $6.665 \mathrm{E}-03$ & $6.714 \mathrm{E}-04$ & $3.063 \mathrm{E}-05$ \\
Point 2 & After & $6.540 \mathrm{E}-03$ & $5.733 \mathrm{E}-04$ & $2.633 \mathrm{E}-05$ \\
\hline Design & Before & $7.605 \mathrm{E}-03$ & $7.356 \mathrm{E}-04$ & $2.878 \mathrm{E}-05$ \\
Point 3 & After & $7.471 \mathrm{E}-03$ & $6.643 \mathrm{E}-04$ & $2.543 \mathrm{E}-05$
\end{tabular}

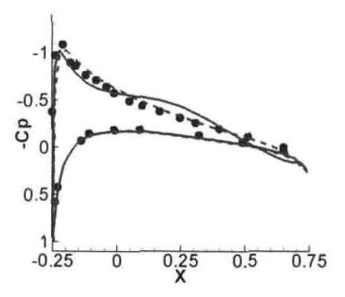

a) $r / R=0.82$

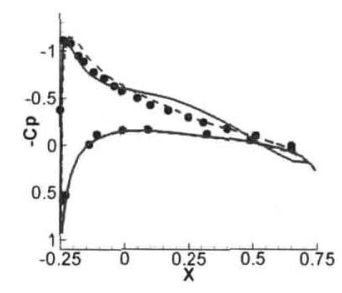

b) $r / R=0.87$



c) $r / R=0.92$

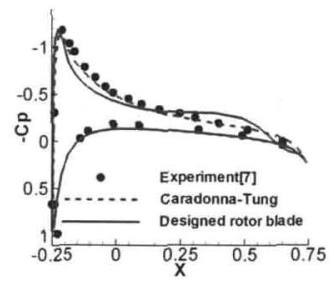

d) $r / R=0.97$

Fig. 10. Comparison of the surface pressure distributions at an off-design subsonic tip Mach number

\section{UH-60 rotor blade}

The second application of the present method was made to the optimal design of the $\mathrm{UH}-60$ helicopter rotor blade [8]. The $\mathrm{UH}-60$ rotor has four twisted blades with an aspect ratio of 15.31, and the blades are composed of two sets of airfoil sections, SC1095 and SC1094R5. The blade planform has a backward sweep of 20 degrees at the tip region.

The UH-60 rotor blade experiences several different flows depending on the flight condition. In hover, the blade operates at a subsonic tip Mach number of 0.65 . At the cruise forward flight, the tip Mach number at the advancing side of the rotor reaches to 0.85 at the collective pitch angle of 5.5 degrees. At the maximum level flight, the helicopter flies at a freestream Mach number of 0.24 , and the maximum tip Mach number at the advancing side becomes 0.89 .

The design points chosen for the present multi-point optimization study were combinations of high tip Mach number and low collective pitch angle, and low Mach number and high collective pitch angle. These design points are; i) tip Mach number 0.65 and 6.5 degree collective pitch angle, ii) tip Mach number 0.85 and 5.5 degree collective pitch angle, and iii) tip Mach number 0.89 and 4.5 degree collective pitch angle.

Figure 11 shows the surface triangulations on the rotor blade. In the present calculation, the far field boundary was located at five radii away from the rotor in all directions. The computational domain was initially divided by 575,940 tetrahedral cells and 109,573 nodes. After applying three levels of solution-adaptive mesh refinement, the number of cells and nodes increased to 668,245 and 125,563 . The mesh adaptation sequence for the second design point is presented in Fig. 12. 


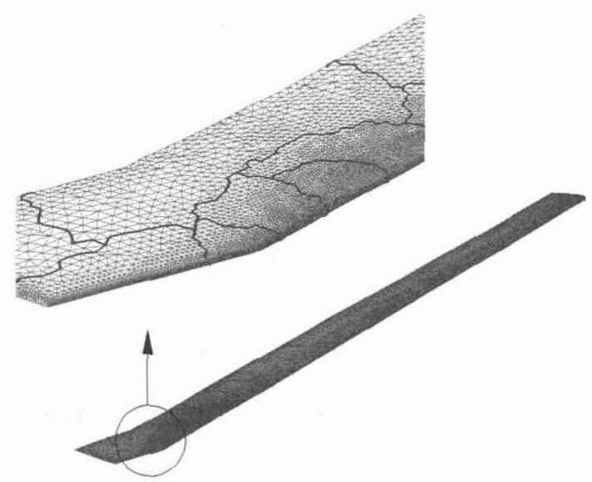

Fig. 11. Surface triangulations on the $\mathrm{UH}-60$ rotor blade

For the aerodynamic shape optimization, nine spanwise stations were selected as the design sections, mostly at the outboard region of the blade $(r / R=0.65,0.70,0.75,0.80$, $0.85,0.90,0.93,0.95,0.98)$. Ten Hicks-Henne shape functions were used at each design section, and thus 90 design variables were used in total.

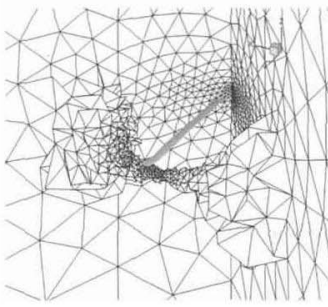

a) First-level adaptation

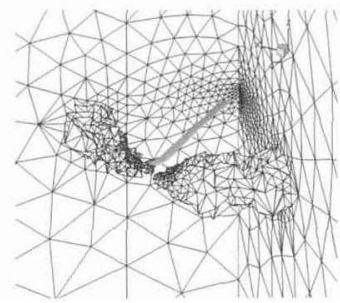

b) Second-level adaptation

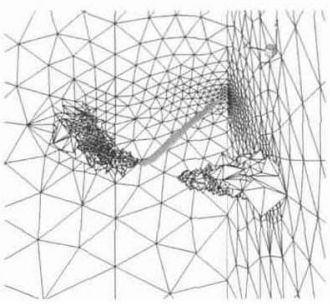

c) Third-level adaptation

\begin{tabular}{|c|c|c|}
\hline & ncell & nnode \\
\hline Initial & 575,940 & 109,573 \\
\hline 1 level & 589,466 & 111,942 \\
\hline 2 level & 627,122 & 118,429 \\
\hline 3 level & 668,245 & 125,563 \\
\hline
\end{tabular}

Fig. 12. Wake mesh adaptation sequence for the $\mathrm{UH}-60$ rotor

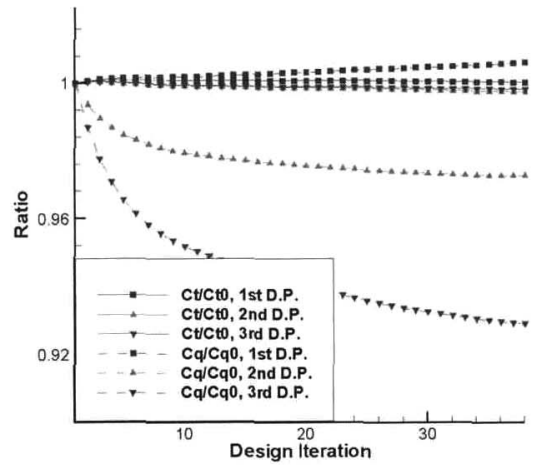

Fig.13. Design convergence history of the $U H-60$ rotor blade

In Fig. 13, the design history of the UH-60 rotor blade is presented. After 35 design iterations, the inviscid torque was reduced up to $8 \%$ while less than $1 \%$ of the thrust variation was observed at all design points. Since the reduction in torque for inviscid transonic flows is achieved by reducing the shock wave strength, the torque reduction was observed mostly for the second and third design points where strong shock waves exist on the blade upper surface. Not much improvement was achieved for the first design point with a subsonic tip Mach number. 


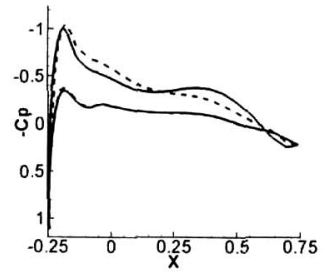

a) $r / R=0.82$

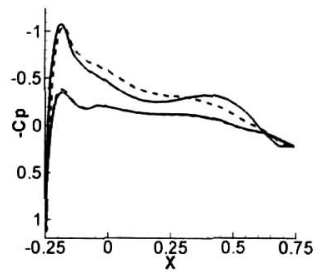

b) $r / R=0.87$

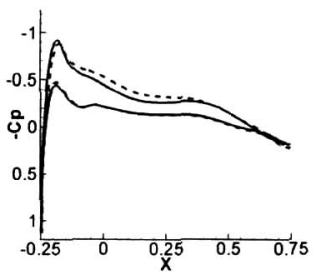

c) $r / R=0.92$

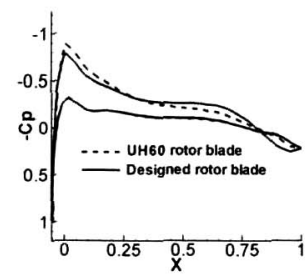

d) $r / R=0.97$

Fig. 14. Comparison of the surface pressure distributions for the first design point.

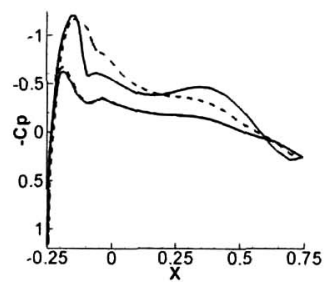

a) $r / R=0.82$

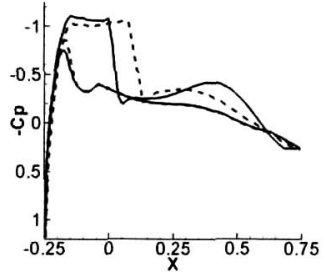

b) $r / R=0.87$

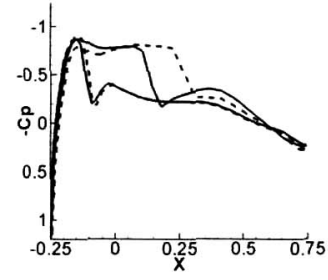

c) $r / R=0.92$



d) $r / R=0.97$

Fig. 15. Comparison of the surface pressure distributions for the second design point

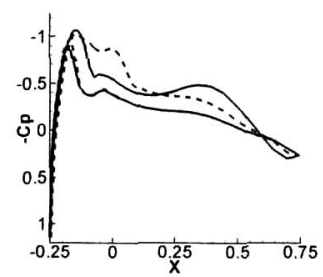

a) $r / R=0.82$

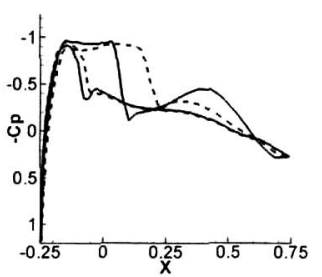

b) $r / R=0.87$

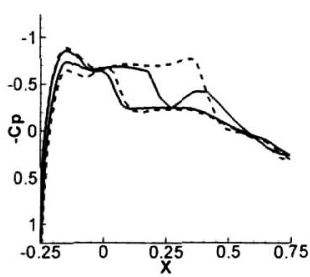

c) $r / R=0.92$

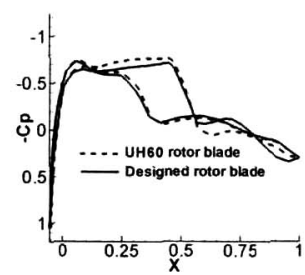

d) $r / R=0.97$

Fig. 16. Comparison of the surface pressure distributions for the third design point

In Figs. 14, 15, and 16, the surface pressure distributions on the UH-60 rotor blade before and after the shape optimization are presented for each design point. Again, after the design optimization, the transonic shock wave strength at the blade tip region is significantly reduced as shown in Figs. 15 and 16.

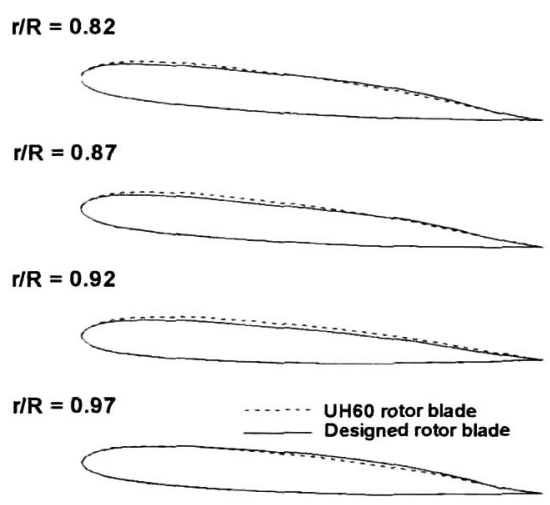

Fig. 17. Comparison of the UH-60 blade section contours at selected spanwise stations 
In Fig. 17, the blade section contours at four spanwise stations near the blade tip are compared between the original and designed rotors. It shows that the leading-edge radius and the blade thickness ahead of the mid chord were slightly reduced for all design sections. At the downstream of the mid chord, the blade thickness increased slightly, and this tendency is typically known for inviscid flow optimizations.

Table 2. Comparison of aerodynamic coefficients of the UH60 rotor blade before and after design

\begin{tabular}{|l|c|ccc|}
\hline \multicolumn{2}{|l|}{} & $\mathrm{Ct}$ & $\mathrm{Cq}$ & $\mathrm{Cm}$ \\
\hline Design & Before & $3.596 \mathrm{E}-03$ & $2.529 \mathrm{E}-04$ & $-1.706 \mathrm{E}-05$ \\
point 1 & After & $3.597 \mathrm{E}-03$ & $2.545 \mathrm{E}-04$ & $-1.744 \mathrm{E}-05$ \\
\hline Design & Before & $3.271 \mathrm{E}-03$ & $2.338 \mathrm{E}-04$ & $-1.810 \mathrm{E}-05$ \\
point 2 & After & $3.262 \mathrm{E}-03$ & $2.273 \mathrm{E}-04$ & $-1.833 \mathrm{E}-05$ \\
\hline Design & Before & $2.426 \mathrm{E}-03$ & $1.847 \mathrm{E}-04$ & $-2.113 \mathrm{E}-05$ \\
point 3 & After & $2.421 \mathrm{E}-03$ & $1.716 \mathrm{E}-04$ & $-1.985 \mathrm{E}-05$ \\
\hline
\end{tabular}

In table 2, the thrust, torque, and pitching moment of the UH-60 rotor blade are compared before and after the design optimization. The result shows that the designed rotor blade exhibits thrust and moment characteristics equivalent to those of the original rotor blade, while more reduction in the required torque was obtained as the flight tip Mach number increases.

In order to check the off-design performance of the designed rotor blade, the aerodynamic performance was evaluated at a subsonic tip Mach number of 0.628 and a collective pitch angle of 10.47 degrees. In Fig. 18, the blade surface pressure distributions before and after the design optimization are compared. It shows that the pressure distributions of the designed blade are similar to those of the original rotor blade for all sections. However, the suction peak at the leading edge is slightly reduced due to the change in the leading-edge radius.

Table 3. Off-design performance of the designed rotor blade

\begin{tabular}{|c|cc|}
\hline & Thrust & Moment \\
\hline UH60 rotor blade & $7.498 \mathrm{E}-03$ & $-2.702 \mathrm{E}-05$ \\
Designed rotor blade & $7.877 \mathrm{E}-03$ & $-2.694 \mathrm{E}-05$ \\
\hline
\end{tabular}

In table 3 , the aerodynamic coefficients of the blade at the off-design operation condition are compared before and after the design optimization. It shows that the total thrust of the designed blade is approximately $5 \%$ higher than the original blade, while the nose-down pitching moment is almost identical between the two blades. Thus, even though the shape optimization was applied to transonic flight conditions, the designed rotor blade still exhibits good performance equivalent to that of the original blade at subsonic tip Mach numbers.

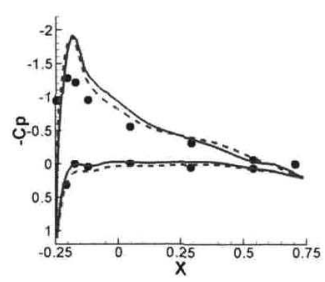

a) $r / R=0.82$

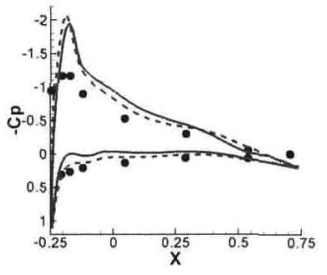

b) $r / R=0.87$

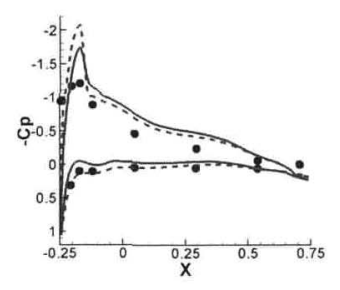

c) $r / R=0.92$

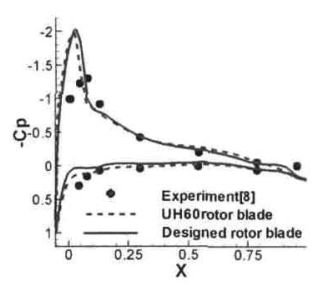

d) $r / R=0.97$

Fig. 18. Comparison of the surface pressure distributions at an off-design subsonic tip Mach number 


\section{Concluding Remarks}

In the present study, a multi-point aerodynamic shape optimization technique has been developed for helicopter rotor blades in hover based on a continuous adjoint method on unstructured adaptive meshes. For this purpose, the flow solver and the adjoint sensitivity analysis were cast on the rotating frame of reference. The blade section contour was modified by using the Hicks-Henne shape functions. A spring analogy technique was adopted to properly handle the mesh movement due to the change of the blade geometry definition. A parallel computation algorithm based on domain decomposition was adopted for the efficient evaluation of the flow and adjoint solvers. The present method was applied to the aerodynamic shape optimization of the Caradonna-Tung rotor blade and the $\mathrm{UH}-60$ rotor blade. It was demonstrated that the present multi-point aerodynamic shape optimization technique is an effective and useful tool for the determination of optimum helicopter rotor blade geometries by significantly reducing the required torque while properly maintaining the desired thrust level.

\section{Acknowledgments}

This study has been supported by the KARI under KHP Dual-Use Component Development Program funded by the MOCIE.

\section{References}

1. Lee, S. W., and Kwon, O. J., "Parallel 3-D Aerodynamic Shape Optimization on Unstructured Meshes," KSAS International Journal, Vol. 4, No. 1, 2003, pp. 45-52.

2. Jameson, A., and Vassberg, J. C., "Computational Fluid Dynamics for Aerodynamic Design : Its Current and Future Impact," AIAA-2001-0538, 2001.

3. Tapia, F., Sankar, L. N., and Schrage, D. P., "An Inverse Aerodynamic Design Method for Rotor Blades," Journal of the American Helicopter Society, Vol. 42, No. 4, 1997, pp. 321-326.

4. Sun, J. Kim, Y., Lee, S., and Lee, D., "Aerodynamic Design of Helicopter Rotor Blade in Forward Flight Using Response Surface Methodology," American Helicopter Society $58^{\text {th }}$ Annual Forum, 2002.

5. Lee, S. W., and Kwon, O. J., "Aerodynamic Shape Optimization of Hovering Rotor Blades in Transonic Flow Using Unstructured Meshes," AIAA Journal, Vol. 44, No. 8, 2006.

6. Lee, S. W., and Kwon, O. J., "2-D Robust Design Optimization on Unstructured Meshes," $5^{\text {th }}$ Asian Computational Fluid Dynamics, 2003.

7. Caradonna, F. X., and Tung, C., "Experimental and Analytical Studies of a Model Helicopter Rotor in Hover," NASA TM 81232, 1981.

8. Ahmad, J. U., and Strawn, R. C., "Hovering Rotor and Wake Calculations with an Overset-Grid Navier-Stokes Solver," American Helicopter Society $55^{\text {th }}$ Annual Forum, 1999.

9. Kang, H. J., Kwon, O. J., "Effect of Wake Adaptation on Rotor Hover Simulations Using Unstructured Meshes," Journal of Aircraft, Vol. 38, No. 5, 2001, pp. 868-877.

10. Frink, N. T., "Upwind Scheme for Solving the Euler Equations on Unstructured Tetrahedral Meshes," AIAA Journal, Vol. 30, No. 1, 1992, pp. 70-77.

11. Holmes, D. G., and Cornell, S. D., "Solution of the 2D Navier-Stokes Equations on Unstructured Adaptive Grids," AIAA 89-1932, 1989.

12. Srinivasan, G. R., Raghavan, V., and Duque, E. P. N., "Flowfield Analysis of Modern Helicopter Rotors in Hover by Navier-Stokes Method," Journal of the American Helicopter Society, Vol. 38, No. 3, 1993, pp. 3-13.

13. Hicks, R. M., and Henne, P. A., "Wing Design by Numerical Optimization," Journal of Aircraft, Vol. 15, No. 7, 1978, pp. 407-412.

14. Blom, F. J., "Consideration on the Spring Analogy," International Journal for Numerical Methods in Fluids, Vol. 32, 2000, pp. 647-668. 